\title{
Morphological Considerations of Fish Fin Shape on Thrust Generation
}

\author{
K. Kikuchi ${ }^{1 \dagger}$, Y. Uehara ${ }^{2}$, Y. Kubota ${ }^{1}$ and O. Mochizuki ${ }^{2}$ \\ ${ }^{1}$ Faculty of Science and Engineering, Toyo University, 2100 Kujirai, Kawagoe, 350-8585 Japan \\ ${ }^{2}$ Department of Biomedical Engineering, Toyo University, 2100 Kujirai, Kawagoe, 350-8585 Japan \\ †Corresponding Author Email: kenji@toyo.jp
}

(Received July 31, 2013; accepted January 12, 2014)

\begin{abstract}
In this study, we aimed to determine the relationship between thrust generation and fish fin shape. To compare the effect fin shape had on thrust generation, we categorized the morphological shapes of fish fins into equilateral polygonal shapes. Polygonal fins were used to generate thrust that depended only on shape. These fins were constructed of a hard elastic material to eliminate any influence of shape deformation. A servomotor with a reciprocal rotation moved a fin cyclically, and thrust was experimentally measured using a strain gage system. Thrust tended to be proportional to the inertia moment of a fin, which indicated difficulty with rotation. Moreover, this trend for thrust generation was directly related to the number of apexes of a polygonal fin. The force translated ratio, which was thrust divided by the force required for fin rotation, was evaluated to determine the hydrodynamic characteristics of fins. This finding showed that the force translated ratio of a fin increased with increased movable perimeter length. The greatest thrust was generated by a triangular fin rotated at its apex, which is often seen in general fish tail fins, whereas the hydrodynamic characteristics were the worst in polygonal fins.
\end{abstract}

Keywords: Fish fin shape, Polygonal fin, Thrust generation, Morphology

\section{NOMENCLATURE}

$\begin{array}{ll}A & \text { projected area } \\ A_{\mathrm{f}} & \text { projected area of fin model } \\ C_{\mathrm{d}} & \text { drag coefficient } \\ D & \text { drag } \\ D_{\mathrm{f}} & \text { drag on fin model } \\ F & \text { thrust } \\ F_{\mathrm{f}} & \text { thrust generated by fin model } \\ F_{\mathrm{th}} & \text { theoretical thrust } \\ f & \text { frequency of rotational fin } \\ I & \text { inertia moment } \\ m & \text { mass } \\ m^{\prime} & \text { added mass }\end{array}$

\section{INTRODUCTION}

In fish, the fin shape suitable for generating the thrust for swimming is dependent on the fish's body size, potential power, swimming speed, habitat, etc. A fish must be able to move as quickly as possible when attempting to escape from a dangerous situation or when hunting living organisms. Thus, fish may have evolved specific adaptations such as various fin shapes that were suitable for their particular habitat or biological reasons. The

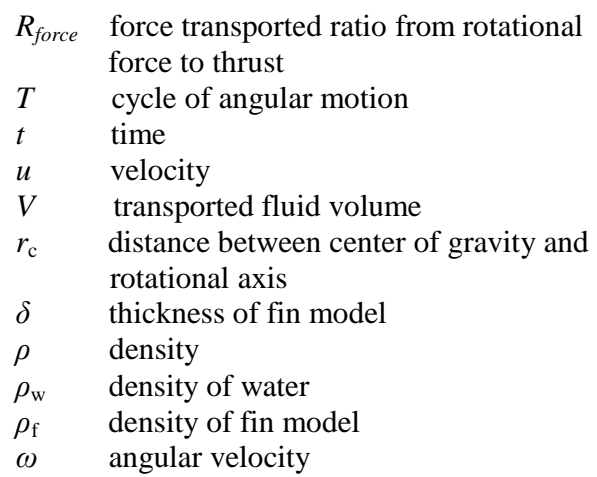

swimming locomotion of common aquatic organisms has been investigated (Azuma 2006; Gray 1936; Lighthill 1960; Lighthill 1969; Sfakiotakis et al. 1999; Vogel 1994). In these studies, swimming speed, body length, frequency of tail fin oscillation, and body drag coefficients were noted as basic variables for continuous swimming. The thrust generation by fish is simply classified into drag-based and lift-based propulsions (Vogel 2003). The remarkably quick motion of fish is generated by an impacting force caused by a 
viscous drag known as drag-based thrust related to vortex generation (Ahlborn et al. 1997). Lift-based thrust is not suited for quick motion, because it is generated in a continuous flow with an appropriate angle of attack of a fin, as observed in large fish with high-speed swimming.

A general fish has a pair of pectoral and ventral fins, an anal fin, a tail fin, and a dorsal fin, as shown in Fig. 1A. The oscillatory locomotion of a tail fin generates thrust by a propulsive flow that is directed backward (Sfakiotakis et al. 1999). A pair of pectoral fins is presumably used to maintain an upright posture when the fish swims continuously by providing a moment of a force around its center of gravity to maintain its balance. In addition, some fish use their pectoral fins for both backward and forward movements during slow swimming by generating drag-based thrust (Drucker and Lauder 2003). Drag-based rather than lift-based thrust is suitable for altitude control, hovering, and rapid turning (Kato 2000; Kato and Liu 2003; Ribak et al. 2010). The dorsal fin is used to stabilize the body against rolling and assists in sudden turns (Drucker and Lauder 2005). Its use is analogous to a vertical stabilizer of an airplane. The roles of other fins remain unclear. Therefore, the tail fin plays an important role in drag-based and lift-based thrusts. Although there are numerous fin shapes in fish, the relationship between thrust and fin shape has not been determined.

Drag- and lift-based propulsions were dependent on the tail fin for thrust generation (Sfakiotakis et al. 1999). Various fish tail fin shapes exist and can be classified based on the symmetrical property of their tops and bottoms (i.e., homocercal and heterocercal types) (Lauder 2000). Both types of these fins were reported to contribute to vortex generation related to thrust. The thrust generated by aquatic organisms has been attributed to the production of a vortex ring using a digital particle image velocimetry (DPIV) technique (Ichikawa and Mochizuki 2008; Kikuchi and Mochizuki 2010; Sakakibara 2004).

The reciprocating motion of the fish tail fin sheds a vortex ring that is directed backward as a reaction of movement. The relationships between thrust and vortex ring, using the PIV technique and the energy balance of motion and vortex circulation were clarified. Recently, the impulsive motion of a rotating fin generated drag-based thrust by vortex ring formation and saturation (DeVoria and Ringuette 2012; Kim and Gharib 2011). The velocity fields of vortex ring generated a jet was measured by using hot-wire anemometry systems (Noro et al. 2013). Although the relationship between thrust generation and vortex shedding has been revealed for the propulsion mechanism by a fin, the contribution of fin shape to thrust generation has not yet been well understood.

A fish robot and a bio-inspired pump that uses a reciprocating fin without a screw propeller has been developed. Although its mechanical mechanism can mimic the locomotion and fin elasticity and shape of an actual fish, the thrust efficiency of this fish robot has not yet achieved that of an actual fish. The optimal shape for the tail fin of this fish robot has not yet been determined, because of the uncertainty of the contribution of tail fin shape to generating thrust. Moreover, the designs of these robots have only been considered for the case of continuous swimming. However, designs for initiating motion or quick motions have not been considered. The fin for a robot fish should be designed based on the specifications of the robot, use conditions, and purposes. Currently, no suitable design exists for fin shape for thrust generation; hence, morphological design should be derived biomimetically from natural fish fins.

In this study, we compared the thrusts generated by rotating polygonal fins that morphologically mimicked fish fin shapes. Elastic plates were used as the fins, because we focused only on thrust generation according to fin shape. The polygonal fins generated thrust differently depending on their shape characteristics. Finally, we evaluated the characteristics of thrust generation by polygonal fins and suggested a suitable design for a robot fish fin according to our morphological considerations.

\subsection{Equation of Motion for Swimming Fish}

Swimming fish generate thrust by fin paddling. They need to overcome a drag, inertia, unsteadiness force for movement. The force balance for fish with an unsteady motion is expressed as follows:

$m \frac{d u}{d t}=F-D-m^{\prime} \frac{d u}{d t}$

where an acceleration of body, $a$, is expressed by $d u / d t$ in Eq. 1. The notation $m$ ' is the added mass due to unsteady motion. Namely, a fish has to generate an additional force to move a mass of water $m$ ' when it moves unsteadily. This is the difference from estimating thrust in the steady state. Thrust is expressed by $F$, and drag is expressed by $D$, which includes shape drag and friction drag in Eq. 1. The effect of drag on a fish's body is expressed by the dynamic pressure as follows:

$$
D=C_{D} \frac{1}{2} \rho u^{2} A
$$

where $C_{D}$ is the drag coefficient when the area $A$ is taken as a wetted surface area of a fish and not a projected frontal area, $\rho$ is fluid density, and $u$ is velocity. Thus, drag includes both shape and friction drag. The value of $C_{D}$ for fish is $0.004-0.015$ (Vogel 1994). Because of the streamlined shape of fish, shape drag is considered to be minimal. Thus, this $C_{D}$ value is regarded as friction drag. However, most of these values were obtained by measurements in a steady flow. The skin-friction drag of fish was summarized (Azuma 2006). The value of $C_{f}$, which is the drag coefficient based on the wetted surface area, ranged from 0.003 for a dolphin to 0.5 for a globefish. These values are larger compared with that of a flat plate with the same surface area of a laminar boundary layer. Drag has generally been 
estimated using Eq. 2, assuming the drag coefficient $C_{D}$

From acceleration, $a$, measured by a motion capture system in a steady flow, thrust is estimated as a summation of $m a$ and the body drag. Moreover, if a fish swims at constant speed, the magnitude of thrust is equal to that of drag because $m a=0$ owing to $a=$ 0 . We have not yet been able to understand the drag for even a basic simple body shape. Nevertheless, researchers have used Eq. 1 to estimate thrust based on observation of freely swimming fish. For example, a platy shown in Fig. 1A swam in a continuous flow channel at $50 \mathrm{~mm} / \mathrm{s}$. Hence, this platy swam at continuous speed with no acceleration, and Eq. 1 was rewritten as $F=D$. This implies that thrust was balanced with the drag acting on the fish's body. Thrust, as estimated by Eq. 2, was approximately $0.80 \mu \mathrm{N}$. Thus, a fish needs to overcome the drag acting on it when swimming in a continuous flow.
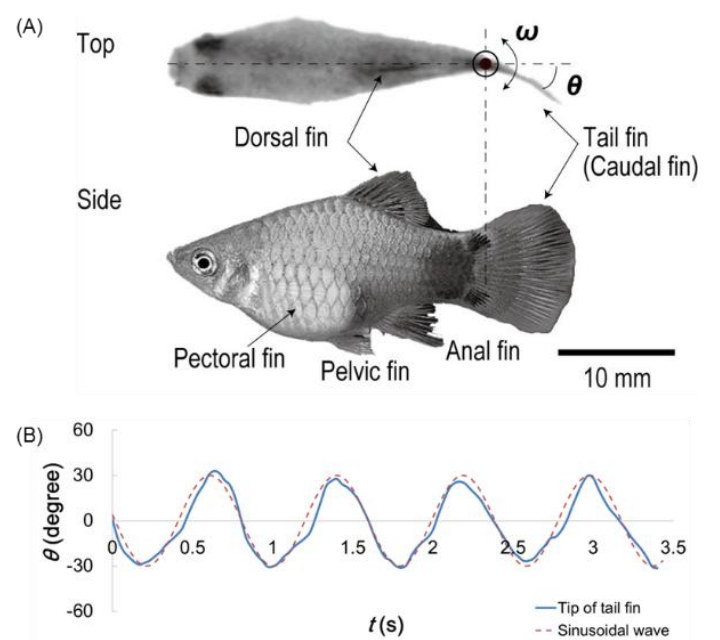

Fig. 1. Platy (Xiphophorus maculatus) (A) Top and side views of a platy. The tail fin was swung at a rotating axis with an angular velocity of $\omega$. (B) The reciprocating motion of the tip of the tail fin when the platy swam in a flow at $50 \mathrm{~mm} / \mathrm{s}$ as compared with a sinusoidal wave with an oscillating frequency, $f$, of $0.8 \mathrm{~Hz}$ and at an angle, $\theta$, of $\pm 30^{\circ}$.

\subsection{Force for Fin Rotational Motion}

In the rotational motion of a fin, the rotational force must be sufficient to overcome inertia and drag. (Azuma 2006) suggested a force balance expression for fin paddling as simple linear motion. To estimate the required force for fin rotation, the rotational force balance must be considered. We rewrote the force balance of a rotating fin into a torque balance between the angular momentum of the oscillating motion of a fish tail fin and drag as follows:

$$
I \frac{d \omega}{d t}=F_{f} r_{c}-D_{f} r_{c}
$$

$$
D_{f}=\frac{1}{2} C_{d r o t} \rho_{w}\left(r_{c} \omega\right)^{2} A_{f}=\frac{C_{d r o t}}{2} \rho_{w} V r_{c} \omega
$$

where $I$ is the inertia moment of a rotating fin, $\omega$ is the angular velocity of the fin, $t$ is time, $F_{f}$ is the force for rotating the fin, $r_{c}$ is distance between the axis of rotation and the center of gravity of the fin, and $D_{f}$ is the drag acting on a fin that was rewritten for rotational conditions using Eq. 2. $C_{d r o t}$ is the rotational drag coefficient, and $A_{f}$ is the projection area of a fin. Equation 4 can be rewritten to include momentum of a transported fluid volume, $V$. In other words, this implies that the fin converts the momentum of fluids to drag. If $C_{d r o t}=2$, the fin converts all the momentum to drag. This implies that drag can be interpreted by its relationship to momentum translation. The angular component in parallel with the swimming direction of rotational force is used as thrust, $F$, in the following equation:

$$
F=F_{f} \sin |\theta|=\left(\frac{C_{d r o t} \rho_{w} V r_{c} \omega}{2}+\frac{I}{r_{c}} \frac{d \omega}{d t}\right) \sin |\theta|
$$

where the angle of the fin, $\theta$, is the angle between the fin cord line and the thrust direction. Equation 5 indicates the instantaneous thrust by a rotating fin. In this paper, the relationship between thrust generation and the morphological shape of a rotating fin is discussed using inertia moment and drag.

\section{MATERIALS AND METHODS}

\subsection{Experimental Apparatus}

The thrust measurement system consisted of a strain gage system, with thrust generation by a rotating fin. This system was installed in a water tank with still water to measure the thrust generated when a fish moved quickly or started from rest. The fin was manipulated by a control system connected to aluminum rods and a servomotor controlled by a $\mathrm{H} 8$ microcomputer. Sinusoidal angular motion was generated by a servomotor for $1.2^{\circ}$ for $10-\mathrm{ms}$ intervals. The fin frequency, $f_{\mathrm{f}}$, and the maximum angle of the fin model, $\theta$, were set at $1 \mathrm{~Hz}$ and $\pm 30^{\circ}$, respectively. The one direction of thrust which was classified the forward force or not was measured by two strain gages glued on the rotating axis connected to the fin. The force in the forward direction was designated as thrust, and the force in the other direction was designated drag. The signals from the strain gages connected to a Wheatstone bridge circuit were amplified by an amplifier (DPM-601B; Kyowa Electronic Instrument Co., Ltd.). A calibration curve for the relationship between the strain gage signals and a given force was confirmed using a spring balance. This system could measure the thrust generated by a fin with a minimum resolution of $0.1 \mathrm{mN}$ every 1 $\mathrm{ms}$ for the force and time. The natural frequency of the control system for the fin model in the water tank was measured by the self-oscillation of this system due to resonance and was found to be 11.5 $\mathrm{Hz}$. 


\subsection{Fin Models}

Hard polygonal plates were used as fin models in our experiments to investigate whether thrust generated depended only the shape characteristics and not deformation due to elasticity. Tables 1 and 2 show the models that had polygonal shapes to mimic fish tail fins. The fin models were constructed using a three-dimensional printer (ZPrinter450; 3D Systems Corp.). These models included a triangle, a square, a pentagon, a hexagon, and a circle. The three-dimensional printer constructed the model using material such as calcium sulfate $\left(\rho_{\mathrm{c}}=1800 \mathrm{~kg} / \mathrm{m} 3\right)$ from computeraided design (CAD) data with a $0.1-\mathrm{mm}$ spatial resolution. The density of this material was comparable with that of fish and bird bones, which are the major structural components of fins and wings, respectively (Gillis 2010; Kawasaki and Weiss 2008). We constructed 10 models of a platy tail fin with area, $A_{f}, 4844 \mathrm{~mm}^{2}$ and thickness, $\delta$, $2.0 \mathrm{~mm}$. In addition, the mass of each model, $m$, was within the same value of $17.5 \mathrm{~g}$. The surface of the fin model was polished till smooth and coated with an acrylic resin spray.

Table 1 Line contact models

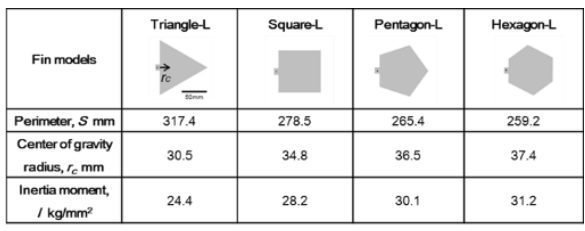

Table 2 Point contact models

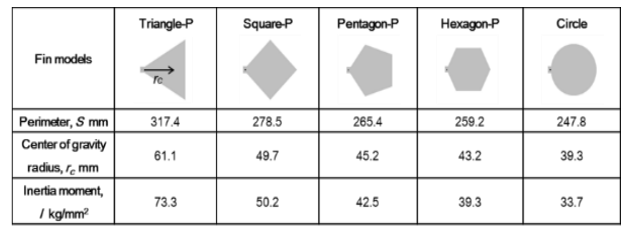

Nine models were classified into two types depending on the connecting position between the model and the rotating rod as a line contact and a point contact as shown in Tables 1 and 2. To avoid confusion between the shape classifications of these models, the models with a line contact with the rod were designated with - $\mathrm{L}$ and others were designated with -P. The inertia moments were estimated by the following equation:

$$
I=I_{0}+\rho_{f} A_{f} \delta r_{c}^{2}
$$

where $I_{0}$ is the inertia moment of the fin model at its center of gravity parallel to the axis of rotation defined as $I_{0}=\int r^{2} d m, \rho_{f}$ is the density of a fin model, and $r_{c}$ is the distance between the axis of rotation and the center of gravity. The total mass of each fin model was the same; hence, the area and thickness were also the same for each model. Therefore, the inertia moment basically tended to increase in direct proportion to the square of $r_{c}$

The blockage ratios related to the distances between the projected widths of the maximum angles of the fin models and the channel widths ranged from 0.19 to 0.25 . These seemed to be quite small compared with a model with an experimental channel with less interference of wall effects from a fundamental experiment (Awbi and Tan 1981). We also confirmed the interference of the side wall of the water tank by constructing a partition board parallel to the side wall close to the tip of the fin model at a maximum angular position. The distance between the tip of the fin model and the wall was approximately $60 \mathrm{~mm}$. The average thrust and the maximum thrust were compared with or without the baffle plate fixed in parallel to the wall at a position that is half the distance between the tip of the fin model and the wall. The thrust with the baffle plate was only $1 \%$ greater than that without it. The effect of blockage was considered to be negligible in our experiments.

\section{RESULTS}

\section{a. Periodical Thrust Generation by Rotating Fish Fin Models}

Figure 2 shows the measured thrusts, $T$, generated by the fin models with the corresponding angle position, angular velocity, and acceleration of the fin models. Phase averaging for 10 cycles and ensemble averaging for 10 samples were applied for each result for the fin models. The absolute velocity with respect to the sinusoidal angular motion of a fin model is shown in Fig. 2B. Thrust was observed during the acceleration phases of $0.25-0.5$ and $0.75-1.0$, when the fin model increased its speed. The maximum velocity of the fin model was reached at an angular position of $0^{\circ}$ when the acceleration of the fin model was zero, and at this point the thrust was nearly zero. Thrusts were periodically generated twice with the frequency oscillation of the fin model. This implied that thrust was generated twice during one cycle.

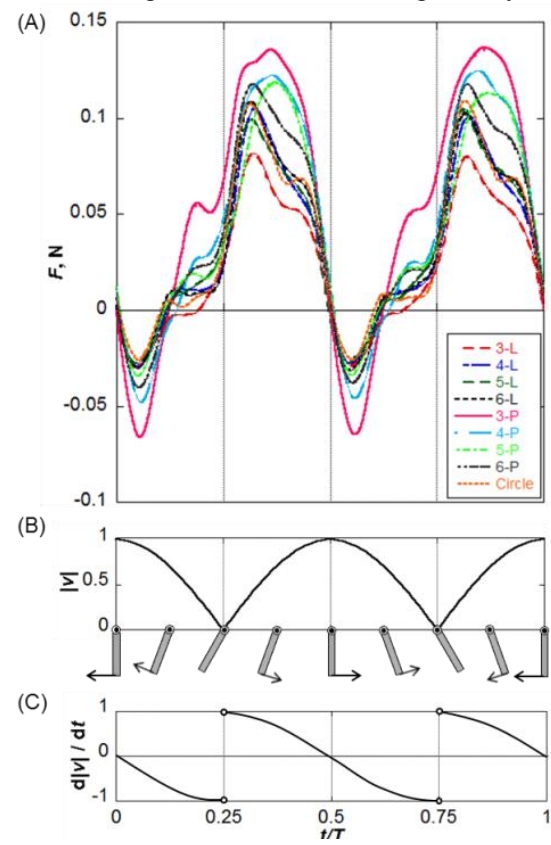

Fig. 2. Changes in thrust generated by the fin models with time. (A) Time history of thrust generation. (B) Angular velocity of a fin model. (C) Acceleration of a fin model. 
The fine oscillations of frequency on the measured thrust were analyzed using the FFT method. These changes in oscillations might have been caused by sympathetic vibrations due to the self-oscillation and natural frequency of the control system of the fin models, because both frequencies indicated a good agreement.

The maximum thrust generated at the maximum angle of the fin model with a delay of $0.07-0.11 \mathrm{~s}$ occurred approximately after the angular position of $12^{\circ}$. In contrast, the minimum thrust, which was work against the drag, was in the opposite direction of the thrust before the angular position of $0^{\circ}$ to $12^{\circ}$. At the angular position near $0^{\circ}$, thrust nearly disappeared. From Eq. 5, it was also suggested that thrust disappeared during this phase because the acceleration was zero. The thrust was comprehensively obtained during the acceleration phase for each fin model. In deceleration phase, the thrust and drag were mixed and shifted at almost half phase.

Figure 3 shows the relationship between the thrust generated in a cycle and the inertia moment of a fin. The time-averaged thrusts in a cycle, $\bar{F}$, were calculated using the Simpson's method from Fig. 2 as $53.8 \pm 2.0,45.4 \pm 1.4,41.9 \pm 1.5,41.3 \pm 0.6$, $34.5 \pm 1.5,33.7 \pm 1.6,33.5 \pm 1.0,32.1 \pm 0.4$, and $22.6 \pm 1.9 \mathrm{~N}$ for Triangle-P, Square-P, Pentagon-P, Hexagon-P, Circle, Hexagon-L, Pentagon-L, Square-L, and Triangle-L, respectively. Experimental values for 10 samples are expressed as mean \pm s.d. These thrusts were in descending order based on the inertia moments of the fin models.

For the models whose axis of rotation was located at a apex, these thrusts increased with decreasing order of polygon vertices. For Triangle-P, which is similar to an ordinary fish's tail fin, both the peak and averaged thrusts were the maximum. The circle model showed minimum peak values compared with the other models. In contrast, the models whose axis of rotation was located at a side, these thrusts increased with an increasing number of vertices. Increasing number of its edge connecting at a side shows tendency to be close the value of circle. Thus, there was an opposite tendency for models connected to the axis of rotation, regardless of whether the thrust increased with an increasing inertia moment. As a result, Triangle-P generated the strongest thrust among these models; in addition, this model had the highest inertia moment.

\section{DISCUSSION}

\section{a. Thrust Generation by Polygonal Fins}

The thrust due to angular fin motion was considered to be generated cyclically according to fin acceleration, because thrust was related to fin acceleration as shown in Fig. 4. The inertia force affected the thrust because of the reaction force that worked in the direction opposite to acceleration. According to fin motion, the flow of fluid was actively induced toward the moving direction of the fin around the fin surface. The drag for a rotating fin depended on the relative velocity between the moving speed of the fin and the surrounding flow. During the acceleration phase, the fin moved against the surrounding flow that occurred due to its axial reverse motion, and hence, the drag effect was in the same direction of thrust. During the deceleration phase, the fin was pushed by the surrounding flow that was induced by the accelerated fin motion, and hence, the drag effect was in the same direction of thrust. The thrust generated by both accelerated and decelerated motion of the fin is indicated by a dashed line in Fig. 4.

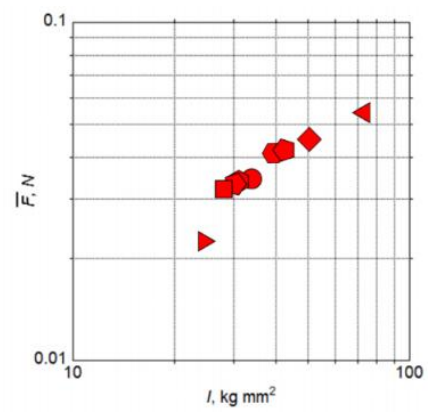

Fig. 3. Experimental averaged thrusts in one cycle vs. inertia moments of fish tail fin models.

In our experimental results, thrust actually did not have a maximum peak at $t / T=0.25$ and 0.75 , which were the delays. Moreover, thrust worked in the opposite direction just after $t / T=0$ and 0.5 . We considered that these delays and drag were caused by the presence of a vortex behind the fin.

In previous research, thrust generation with the accelerated motion of a simple fin model was discussed in relation with vortex formation (Ahlborn et al. 1997; DeVoria and Ringuette 2012; Kim and Gharib 2011). These studies described vortex formation around a fin-like shape during acceleration and deceleration motions. During the deceleration phase, the vortex appeared in front of the model because flow overtook the model. In contrast, during the acceleration phase, the vortex appeared at the back of the model because the model pushed out fluid in the direction opposite to thrust. In our experiments, using a dye to visualize flow, we confirmed that the vortexes were shed around the fin model during acceleration and deceleration motions, as illustrated in Fig. 4. Each surface were painted orange and green dyes at left and right sides and generated clock wise and counter clock wise vortices by fin's rotation respectively.

The drag and thrust induced when the vortex was close to the fin's surface would immediately appear after the acceleration was zero or would be the maximum after a delay related to the growth of the vortex. Once the vortex moved away from the fin's surface, drag and thrust disappeared. The intensity of the vortex or circulation was considered to relate to the volume of fluid transported by the fin motion and the length of the leading edge of a fin model because the circulation around a fin related directly a thrust according as Kutta-Joukowski theorem. 
The duration of the vortex would depend on its intensity. The time sequential effect of the vortex on thrust was considered for the growth and shedding of the vortex, represented as dotted lines in Fig. 4. This broken line shows the thrust component of inertial force generated by rotational fin, which depends on the acceleration of fin motion. Dotted line shows the predictive force by generated vortices, which was calculated by the differences between experimental thrust and the thrust component of inertial force. The predicted thrust that incorporated the acceleration motion and vortex shedding is shown as a solid line in Fig. 4. Both the maximum and minimum peaks for thrust and drag in Fig. 2 might correspond to those of the predicted thrust due to the effect of vortex formation. According to the intensity and shape of a vortex around the rotational fin model, the amplitude and position of the peak would change.

\section{b. Force Translation by a Fish Tail Fin}

A fin obtains the thrust force by a reaction of motion of the surrounding fluid induced by a rotating fin. The rotating fin translates the force for fin rotation to thrust. In drag-based thrust generation, thrust is related to drag, which is based on a momentum transfer theory (Schlichting 1979). The fluids surrounding a rotating fin are pushed out at a velocity corresponding to the fin velocity, and subsequently, this enabled the fin to obtain drag as thrust. The theoretical thrust generated by a rotating fin wasestimated by a momentum theory expressed as follows with $C_{d r o t}=2$ in Eq. 5:

$$
\begin{aligned}
& \bar{F}_{t h}=\int\left(\rho_{w} V r_{c} \omega+\frac{I}{r_{c}} \frac{d \omega}{d t}\right) \sin |\theta| d \theta \\
& R_{\text {force }}=\frac{\bar{F}}{\overline{F_{\text {th }}}}
\end{aligned}
$$
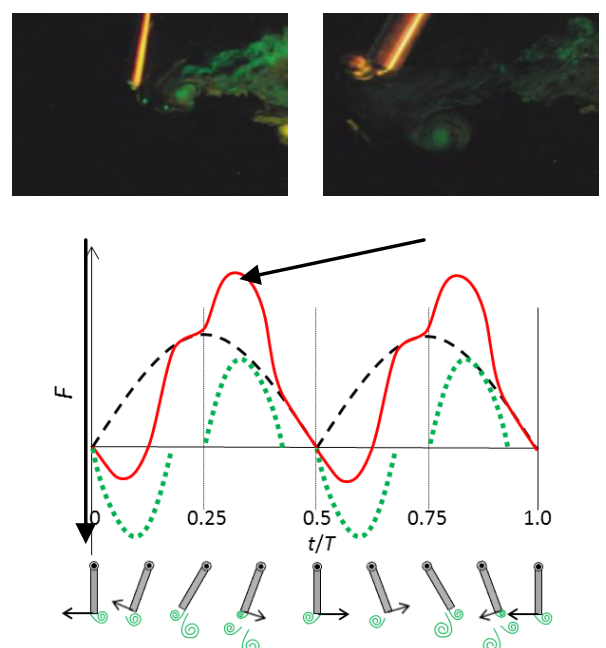

Fig. 4. Thrust generation as a function of angular motion of a fin model and vortex shedding. Arrows indicate the direction of force taken on the fin models. Photographs show the visualization of shedding vortices at the maximum and minimum thrust generating.

Green dye painted on the left side of fin's surface, Orange dye painted on the right side of that.
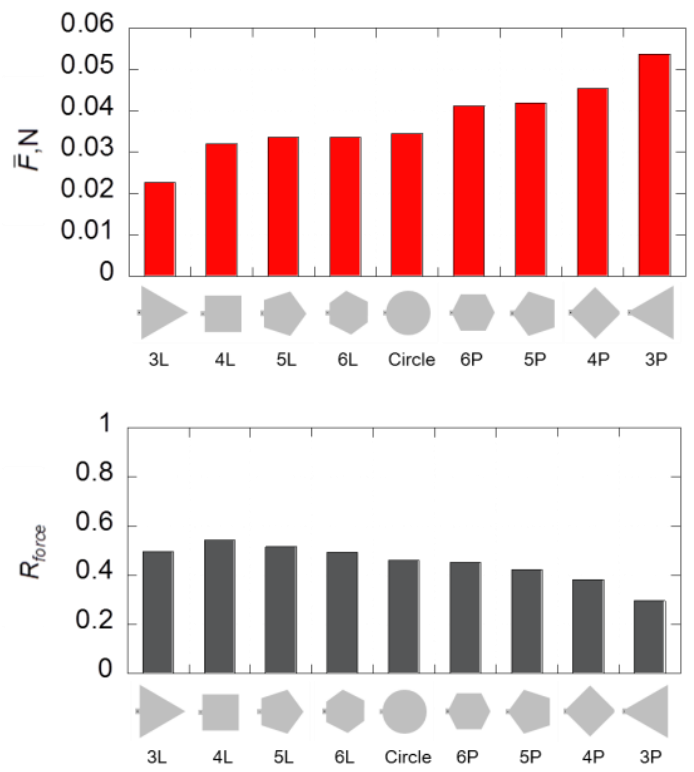

Fig. 5. Comparisons of thrust generation, force translated ratios, and fin models.

Here, $F_{\text {th }}$ is the time-averaged theoretical thrust during a cycle, $\rho_{w}$ is the density of water, $V$ is the transported volume of fluid, $r_{c}$ is the distance between the axis of rotation and the center of gravity of a fin, and $\omega$ is the angular velocity. The force ratio, $R_{\text {force }}$, is the force transported ratio of the force for fin rotation divided by the experimentally measured thrust with fin models. This implies that if all of the transported volume of fluid was ejected in a direction opposite to thrust, this momentum would be completely used for thrust generation, which was as follows: $R_{\text {force }}=1$.

Figure 5 shows the relationships between the averaged thrust; $\overline{F_{\text {th }}}$, the transported ratio; $R_{\text {force }}$, and the number of apexes of a rotating polygonal fin in our experimental results. With the line contact (-L) models, thrust increased with an increasing number of apexes. The movable perimeter length in this model involved one of the sides, because we used a side for rotating axis. In contrast, with the point contact (-P) models, thrust increased with a decreasing number of apexes. Thus, thrust showed a tendency to be proportional to the increasing movable perimeter length and the distance from the axis of rotation to the center of gravity of a fin. The transported volume of fluid is geometrically related to the distance from the rotational axis to the center of gravity hence the rotated polygonal fin with the constant area could transport much fluid by its rotation with increasing the distance. Interestingly these distances have a proportion with the movable perimeter lengths of equilateral polygons.

With decreasing movable perimeter lengths, the transported ratios were $0.498,0.543,0.516,0.494$, $0.459,0.454,0.422,0.381$, and 0.296 . In our experimental results, approximately $30-55 \%$ of the fluid transported by the fin models was used for thrust by ejecting fluids in the direction opposite to 
thrust, while other fluid was ejected to the other direction and did not contribute to thrust generation. The line contact (-L) models had a relatively high momentum for transported ratios. Thrust efficiency tended to decrease with an increasing inertia moment of the fin models, except for Triangle-L. Triangle-L should have had the highest efficiency based on this tendency, but it did not follow this trend. We consider that this phenomenon was due to the presence of the vortex behind the fin and could be revealed by visualizing flow using the PIV technique. The transported ratios of polygonal fins typically showed that the farther the center of gravity of the fin model from the axis of rotation, the lower the effective thrust. This result indicated that the Triangle-L, Square-L, Pentagon-L, and Hexagon-L models could effectively use the transported fluid for thrust. In particular, Square-L was the most energy saving shape for thrust generation with a polygonal fin.

Natural fish fins have various shapes that might acquire optimal shapes according to their environments. For example, fish living in reefs, where the flow is moderate, stay in these reefs to hide from their predators and hence do not require continuous migration. Therefore, the shape of their fins should be suitable for quick motion. These include small and large sized fish that have triangular tail fins. There are two possible reasons for this finding. Small fish will experience negligible effects of inertia, whereas large fish have sufficient power to overcome fin drag. Mediumsized fish that do not have sufficient power against fin drag and would require a different efficient tail fin shape. Square, pentagon, and hexagon fin shapes are commonly observed in the appendage or some fins (e.g., duck and ornithorhynchus). These shapes might be useful for thrust generation during paddling. In contrast, a triangular shaped fin model is observed in general fish tail fins, which not only had low efficiency but also had the greatest inertia moment in our experiments. We found that square, pentagon, and hexagon fin shapes were suitable for drag-based thrust generation and efficiently saved power.

\section{CONCLUSION}

Thrust generation by equilateral polygonal fin models with angular oscillations was experimentally measured to determine the contribution of a fish fin's morphological shape. Polygonal fins that mimicked fish fins generated thrust differently depending on their shapes. There was a tendency for increased thrust generation by fin models with increasing inertia moment. We also evaluated thrust efficiency using theoretical thrust generated according to a momentum theory. Theoretical thrust showed a trend that was in good agreement with our experimental results. Moreover, thrust increased with increasing inertia moment for a fin model, whereas the thrust translated ratio decreased. As a result, the timeaveraged thrust generated by a fin increased with an increasing inertia moment in the same rotational motion. There was also a geometric tendency between thrust generation and the number of apexes of a polygonal fin. The greatest thrust was generated by a triangle fin rotated at an apex, which is often seen in general fish tail fins, whereas the hydrodynamic characteristics were worst in polygonal fins. For the design of fin shape's fish robot which has a strong thrust generation, the triangle shape rotated on the apex is the best suitable shape.

\section{REFERENCES}

Ahlborn B, Chapman S, Stafford R, Harper R. (1997). Experimental simulation of the thrust phases of fast-start swimming of fish. Journal of Experimental Biology 200(17), 2301-2312.

Awbi HB, Tan SH. (1981). Effect of Wind-Tunnel Walls on the Drag of a Sphere. Journal of Fluids Engineering 103(3), 461-465.

Azuma A. (2006). Swimming by Fanning: American Institute of Aeronautics and Astronautics. 313-386.

DeVoria AC, Ringuette MJ. (2012). Vortex formation and saturation for low-aspect-ratio rotating flat-plate fins. Experiments in Fluids 52(2), 441-462.

Drucker EG, Lauder GV. (2003). Function of pectoral fins in rainbow trout: behavioral repertoire and hydrodynamic forces. Journal of Experimental Biology 206(5), 813-826.

Drucker EG, Lauder GV. (2005). Locomotor function of the dorsal fin in rainbow trout: kinematic patterns and hydrodynamic forces. Journal of Experimental Biology 208(23), 4479-4494.

Gillis GB. (2010). Weing in on bird bones. The Journal of Experimental Biology 213(17):v-v.

Gray J. (1936). Studies in Animal Locomotion: VI. The Propulsive Powers of the Dolphin. Journal of Experimental Biology 13(2), 192199.

Ichikawa S, Mochizuki O. (2008). The Flow Induced by a Jellyfish. Journal of Visualization 11(3), 257-264.

Kato N. (2000). Control performance in the horizontal plane of a fish robot with mechanical fins. IEEE Journal of Oceanic Engineering. 25, 121-129.

Kato N, Liu H. (2003). Optimization of motion of a mechanical pectoral fin mechanical systems, Machine Elements and Manufacturing 46(4), 1356-1362.

Kawasaki K, Weiss KM. (2008). SCPP gene evolution and the dental mineralization continuum. Journal of Dental Research. United States., 520-531. 
K. Kikuchi et al. / JAFM, Vol. 7, No. 4, pp. 625-632, 2014.

Kikuchi K, Mochizuki O. (2010). Consideration of thrust in escaping motion of a mosquito larva. Journal of Aero Aqua Bio-mechanisms 1(1), 111-116.

Kim D, Gharib M. (2011). Characteristics of vortex formation and thrust performance in dragbased paddling propulsion. The Journal of Experimental Biology 214(13), 2283-2291.

Lauder GV. (2000). Function of the Caudal Fin During Locomotion in Fishes: Kinematics, Flow Visualization, and Evolutionary Patterns. American Zoologist 40(1):101-122.

Lighthill MJ. (1960). Note on the swimming of slender fish. Journal of Fluid Mechanics 9(02), 305-317.

Lighthill MJ. (1969). Hydromechanics of Aquatic Animal Propulsion. Annual Review of Fluid Mechanics 1(1), 413-446.

Noro Sh, Suzuki Y, Shigeta M, Izawa S and Fukunishi Y (2013). Boundary Layer Receptivity to Localized Disturbances in Free stream Caused by a Vortex Ring Collision.
Journal of Applied Fluid Mechanics 6(3), 425433

Ribak G, Swallow JG, Jones DR. (2010). DragBased 'Hovering' in Ducks: The Hydrodynamics and Energetic Cost of Bottom Feeding. PLoS ONE 5(9):e12565.

Sakakibara. (2004). Stereo-PIV study of flow around a maneuvering fish. Experiments in fluids 36(2):282.

Schlichting H. (1979). Boundary-layer theory. New York: McGraw-Hill. xxii, 817.

Sfakiotakis M, Lane DM, Davies JBC. (1999). Review of fish swimming modes for aquatic locomotion. IEEE Journal of Oceanic Engineering, 24(2):237-252.

Vogel S. (1994). Life in moving fluids : the physical biology of flow. Princeton, NJ: Princeton University Press. xiii, 467

Vogel S. (2003). Comparative biomechanics : life's physical world. Princeton, NJ. Princeton University Press. xii, 580 